\title{
Upper extremity injuries in the victims of intimate partner violence
}

\author{
Richard Thomas ${ }^{1} \cdot$ George S. M. Dyer ${ }^{2} \cdot$ Paul Tornetta $\mathrm{III}^{3} \cdot$ Hyesun Park ${ }^{1} \cdot$ Rahul Gujrathi $^{1} \cdot$ Babina Gosangi $^{1}$. \\ Jordan Lebovic ${ }^{4}$. Najmo Hassan ${ }^{1} \cdot$ Steven E. Seltzer ${ }^{1} \cdot$ Kathryn M. Rexrode $^{5} \cdot$ Giles W. Boland $^{1} \cdot$ Mitchel B. Harris $^{6}$. \\ Bharti Khurana ${ }^{1}$ (10)
}

Received: 30 August 2020 / Revised: 1 November 2020 / Accepted: 23 December 2020 / Published online: 18 January 2021

(C) European Society of Radiology 2021

\begin{abstract}
Objectives To recognize most common patterns of upper extremity (UE) injuries in victims of Intimate Partner Violence (IPV). Methods Radiological review of 308 patients who reported physical IPV at our institution from January 2013 to June 2018, identified 55 patients with 88 unique UE injuries. Demographic data and injury patterns and associations were collected from the electronic medical records.

Results The cohort included 49 females and 6 males (age 19-63, mean 38). At the time of injury, IPV was reported in 15/88 $(17 \%)$ and IPV screening was documented for 22/88 (25\%) injuries. There were 46 fractures, 8 dislocations or subluxations, and 34 isolated soft tissue injuries, most commonly involving the hand (56/88). Fractures most commonly involved the fingers (21/ $46,46 \%)$ and the 5 th digit $(8 / 27,30 \%)$. Medial UE fractures (5th digit, 4 th digit) constituted $44 \%$ of hand and finger fractures (12/ $27)$ and $26 \%$ of all fractures (12/46). Comminuted and displaced fractures were rare $(8 / 46,17 \%)$. Head and face injuries were the most common concomitant injuries $(9 / 22,41 \%)$ and subsequent injuries $(21 / 61,35 \%)$. Of 12 patients with recurrent UE injuries, 6 had recurrent injuries of the same hand. Five of 6 non-acute fractures $(83 \%)$ were of the hand.

Conclusions Hand and finger injuries are the most common UE injuries in patients with IPV, with finger being the most common site and medial hand the most common region of fracture. Repeated injuries involving the same site and a combination of medial hand and head or face injuries could indicate IPV.

Key Points

- Upper extremity injuries in victims of intimate partner violence are most commonly seen in the hand and fingers.

- Fingers are the most common site of fracture and the medial hand is the most common region of fracture in the upper extremity in victims of intimate partner violence.

- In intimate partner violence victims with upper extremity injuries, concomitant injuries and subsequent injuries are most commonly seen in the head and neck region.
\end{abstract}

Keywords Intimate partner violence $\cdot$ Domestic violence $\cdot$ Abuse $\cdot$ Upper extremity $\cdot$ Radiology

Summary statement In victims of intimate partner violence, fractures most commonly involve the fingers, with head and neck being the most common site of concomitant and subsequent injuries.

Bharti Khurana

bkhurana@bwh.harvard.edu

1 Department of Radiology, Division of Emergency Radiology, Brigham and Women's Hospital, Harvard Medical School, 75 Francis Street, Boston, MA 02115, USA

2 Department of Orthopedic Surgery, Brigham and Women's Hospital, Harvard Medical School, 75 Francis Street, Boston, MA 02115, USA
3 Department of Orthopedic Surgery, Boston Medical Center, 725 Albany St 4th Floor, Suite 4B, Boston, MA 02118, USA

4 Department of Orthopedic Surgery, Hospital for Joint Diseases, 301 E 17th St, New York, NY 10003, USA

5 Division of Women's Health, Brigham and Women's Hospital, Harvard Medical School, 75 Francis Street, Boston, MA 02115, USA

6 Department of Orthopedic Surgery, Massachusetts General Hospital, 55 Fruit St, Boston, MA 02114, USA 


$\begin{array}{ll}\text { Abbreviations } \\ \text { COVID-19 } & \text { Coronavirus disease 19 } \\ \text { CT } & \text { Computed tomography } \\ \text { ED } & \text { Emergency department } \\ \text { EHR } & \text { Electronic health record } \\ \text { HIPAA } & \text { Health Insurance Portability and } \\ & \text { Accountability Act } \\ \text { IPV } & \text { Intimate partner violence } \\ \text { MRI } & \text { Magnetic resonance imaging } \\ \text { UE } & \text { Upper extremity }\end{array}$

\section{Introduction}

Approximately one-third of women worldwide experience some form of physical or sexual violence by their intimate partners, known as intimate partner violence (IPV), during their lifetime [1]. Likewise, in the USA, over 1 in 3 women and 1 in 10 men experience contact sexual violence, physical violence, or stalking during their lifetime [2]. The immediate risk of physical injury and death from IPV is associated with many health consequences, including psychiatric, endocrine, musculoskeletal, gynecologic, and reproductive illnesses [3]. Furthermore, identifying victims of IPV is exceptionally challenging as only about $2.5-15 \%$ of individuals with a history of IPV report it to an authority [4].

The challenges in detecting IPV are due to a combination of factors. Physician factors include lack of awareness, scarcity of time during each encounter, inadequate training to screen for and identify IPV, and reluctance to broach an uncomfortable topic [5]. Patient factors include feelings of shame, fear of consequences, and fear of not being believed [6]. Victims of IPV frequently ascribe their injuries to other causes such as falls, work-related injuries, accidents, for example. Therefore, identifying IPV from a reported history or clinical chart notes alone would not identify all cases of IPV.

In several countries, there has been a recent increase in the utilization of domestic abuse helplines and other resources, in response to the worsening domestic abuse crisis $[7,8]$. This is probably a consequence of the social restrictions and economic stresses incurred by the current coronavirus disease 19 (COVID-19) pandemic [9-11]-victims are now trapped at home with their abusers. Therefore, there is a renewed urgency to identify victims using all available resources.

Traditionally, the role of imaging in trauma has been limited to detecting and characterizing injuries. However, in the setting of IPV, imaging can provide objective data that can serve as the impetus for discussion between the treating clinician (or other providers) and the patient [12, 13]. Emergency department imaging serves a critical role as it has been demonstrated that up to $44 \%$ of victims of homicide related to IPV present to an emergency department within the 2 years preceding the homicide. Over $90 \%$ of these present with an injury
[14]. Identifying characteristic injury patterns can alert physicians to IPV and potentially prevent a more severe injury in the future. Therefore, beginning the discussion about IPV can be a matter of life and death. The head and neck and upper extremity (UE) are the most commonly injured sites in IPV $[13,15]$. While there are previously published articles describing the patterns of head and face injuries in victims of IPV [16, 17], to the best of our knowledge, there is no published data on the patterns of UE injuries in these patients.

Thus, the purpose of our study is to document the upper extremity injuries identified in victims of IPV, identify the most common injury patterns, which can, in turn, serve as a warning sign to treating providers that the patient is at risk.

\section{Materials and methods}

\section{Cohort identification}

This retrospective study was approved by our institutional review board, and it is Health Insurance Portability and Accountability Act (HIPAA)-compliant. The need for informed consent was waived. There was no industry support for our study, and the authors had control of the data and information submitted for publication. We reviewed EHR of all 308 consecutive patients who reported history of physical IPV, to the IPV intervention and prevention program by themselves between January 2013 and June 2018 . Some of these patients were encouraged by their health care providers, while others came across the flyers posted on hospital walls and decided to contact the program. The imaging studies had been ordered at the time of presentation to the emergency department of a level 1 trauma center with trauma. Using our institutional research patient data registry, we excluded 20 patients with no radiological studies. Four radiologists training in emergency radiology fellowship (with 5 years, 6 years, 8 years, and 9 years of experience in radiology) reviewed charts of 288 patients to identify 55 patients with 76 radiological studies demonstrating upper extremity injuries. All 76 radiological studies (72 X-ray, $2 \mathrm{CT}, 2 \mathrm{MRI}$ ) were then reviewed by an emergency radiology fellow with 8 years of experience to identify 88 unique UE injuries. We elected to not include controls in our study because only about $2.5-15 \%$ of individuals with a history of IPV disclose it to an authority [4]. Therefore, defining a true control population is very challenging and is fraught with the possibility of inadvertently including false negative cases.

\section{Data collection}

The demographic information, including age, sex, and race, and medical information including history of substance abuse, 
presence of mental illness, and presence of other comorbidities were collected from the medical record for all 55 patients. The reported mechanism of injury and documentation of screening for IPV at the time of the injury was also recorded.

\section{Imaging finding analysis}

Injuries were categorized as fracture, subluxation/dislocation, and isolated soft tissue injury. The side (right or left) and specific anatomic site of the injury in the upper limb was noted. In case of fracture and subluxation/dislocation, the accompanying adjacent soft tissue swelling was not recorded separately. For studies with multiple injuries, each distinct injury was reported separately. Fractures were described as acute or non-acute, displaced or non-displaced, and comminuted or non-comminuted. The number of patients with recurrent UE injuries at different time points was also noted.

The charts were reviewed for details of other injuriesconcomitant injuries (injuries at the time of the UE injury), injuries after their UE injury, and injuries before their first UE injury. Descriptive statistics for continuous measures are presented as means with standard deviations and as frequencies with proportions for categorical measures.

\section{Results}

\section{Demographics and risk factors:}

The majority of patients were women (49 of 55, 89\%) and were predominantly white $(22,40 \%)$ or African American $(20,36 \%)$. The mean age was 38 years (median 34 years; range 19-63). There was a known psychiatric history in 27 (49\%), a history of substance abuse in 16 (29\%), and other comorbidities in 15 (27\%) patients. The most common comorbidities were chronic musculoskeletal pain or fibromyalgia $(6 / 15,40 \%)$ followed by hypertension $(5 / 15,33 \%)$ and irritable bowel syndrome $(4 / 15,27 \%)$.

\section{Injury reporting and IPV documentation}

The most commonly reported mechanism of injury at the emergency department triage was an assault by an intimate partner $(15 / 88,17 \%)$, followed by a fall $(13 / 88,15 \%)$. However, the reported mechanism of injury was unavailable on chart review for 39 injuries (44\%). IPV screening at the time of injury was documented only in $22 / 88$ injuries (25\%). IPV was documented in the EHR of only 36 patients (65\%) even though all 55 patients subsequently reported IPV to the violence prevention program. In $35 \%$ of patients, there was no formal documentation of IPV in the electronic health records during their visit to the emergency department or later in outpatient clinics, until the timepoint at which the patient self-reported to the institutional
IPV program. Patient demographics and history are summarized in Table 1.

\section{Injury location and pattern}

Among the 55 patients, there were 88 UE injuries, including 46 fractures, 8 dislocations/subluxations, and 34 isolated soft tissue injuries. There were $56(64 \%)$ hand/finger injuries, 26 (29\%) forearm injuries, and $6(7 \%)$ arm/shoulder injuries. Right side upper extremity injuries were slightly more common than left (57\% compared to 43\%) with 17/32 arm/shoulder and forearm injuries, and 33/56 hand/finger injuries on the right side.

\section{Fracture}

The most common location for fracture was the finger (21/46, $46 \%$ ), of which distal phalanges were the most frequent site $(9 / 28,32 \%)$. Overall, finger fractures constituted $46 \%$ of all

Table 1 Demographics, medical, social, and psychiatric history

\begin{tabular}{ll}
\hline & $n=55$ \\
\hline Median age (range) & $34(19-63)$ \\
Gender & \\
Male & $6(11 \%)$ \\
Female & $49(89 \%)$ \\
Race & \\
White & $22(40 \%)$ \\
Black & $20(36 \%)$ \\
Hispanic & $11(20 \%)$ \\
Asian & $1(2 \%)$ \\
American Indian & $1(2 \%)$ \\
Known psychiatric history & Yes $=27(49 \%)$ \\
IPV history & \\
Documented history in chart & Yes $=36(65 \%)$ \\
Screened at time of injury & Yes $=22(40 \%)$ \\
Other comorbidities & Yes $=15(27 \%)$ \\
Substance abuse & Yes $=16(29 \%)$ \\
Recurrent UE injuries & $12(22 \%)$ \\
Recurrent hand injuries of the same side & $6(11 \%)$ \\
Reported MOI & $N=88$ \\
Assault by IP & $15(17 \%)$ \\
Fall & $13(15 \%)$ \\
Assault (not IP) & $8(9 \%)$ \\
Suicide attempt & $2(2 \%)$ \\
Home injury & $3(3 \%)$ \\
No records & $39(44 \%)$ \\
Others & $8(9 \%)$ \\
& \\
\hline &
\end{tabular}

$M O I$ mechanism of injury, IP intimate partner, IPV intimate partner violence, $U E$ upper extremity 
upper extremity fractures in this cohort (21/46). There were 6 metacarpal fractures, and all 6 involved the 5 th digit, including 2 shaft, 2 head or neck, and 1 base (Fig. 1).

The 5 th digit was the most common location (Fig. 2) $(8 / 28$, $29 \%)$ followed by the thumb $(7 / 28,25 \%)$. Combined 5 th and 4 th digit fractures (hypothenar or medial hand fractures) constituted $43 \%$ of hand fractures $(12 / 28)$ and $26 \%$ of all fractures (12/46).

Forearm fractures constituted one-third of all UE fractures $(15 / 46,33 \%)$ (Fig. 3). Radius fractures were twice as common as ulnar fractures.

Comminuted and displaced fractures were rare $(8 / 46$, $17 \%)$, with the majority involving arm or forearm (6/8), including 2 humerus, 2 radius, and 2 ulna. Of 28 hand fractures, there were only 2 comminuted and displaced fractures, both involving phalanges.

Of 88 injuries, there were 6 chronic or non-acute fractures; 5 were of the hand (Fig. 4) and one was of the distal ulna.

\section{Dislocation/subluxation}

The most common location for dislocations/subluxations was the hand $(5 / 8,62 \%)$ with four involving the interphalangeal joints and one involving the metacarpophalangeal joint. The

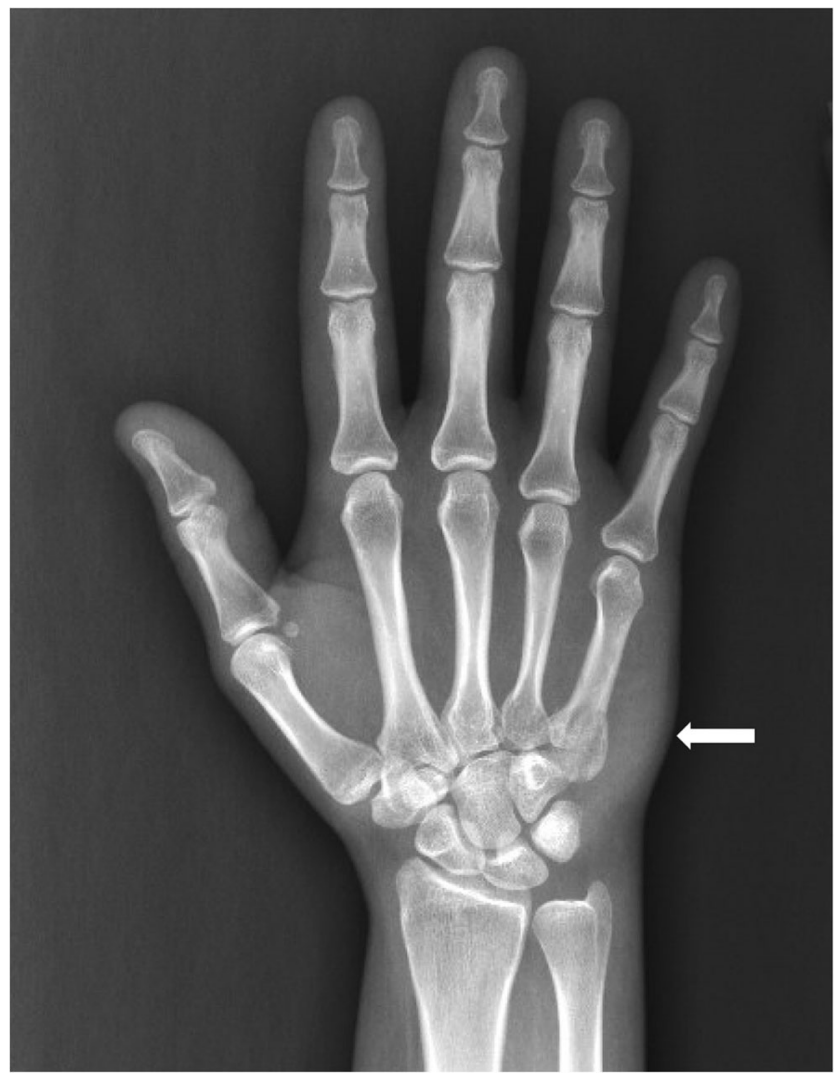

Fig. 1 5th digit metacarpal base fracture: 34-year-old white female with a reported history of fall. Frontal radiograph of the right hand shows a comminuted fracture of the base of the 5th digit metacarpal involving its proximal articular surface (arrow)

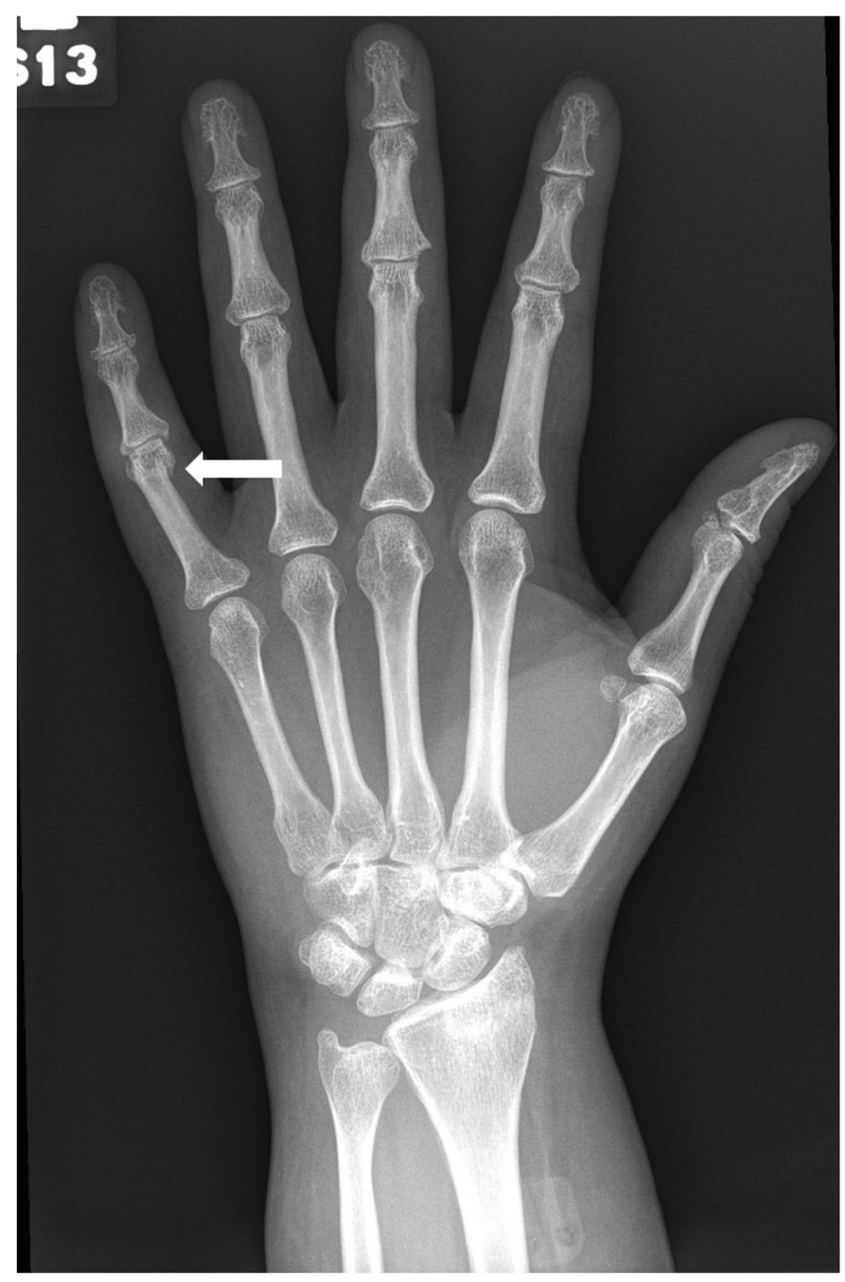

Fig. 2 Medial hand fracture. 43-year-old African American female with a reported history of assault by an intimate partner. Frontal radiograph of the left hand shows an acute fracture of the proximal phalanx of the 5th digit (arrow)

other dislocations/subluxations included one each of the distal radioulnar, acromioclavicular, and elbow joints.

\section{Soft tissue injuries}

The most common location for soft tissue injuries was also the hand $(23 / 34,68 \%)$ followed by the forearm $(9 / 34,26 \%)$. The majority $(32 / 34,94 \%)$ of these were diffuse soft tissue contusions or swelling with one injury of the scapholunate ligament and one of the triangular fibrocartilage complex seen on MRI.

\section{Associated injuries}

Concomitant imaging evidence of injuries was noted in 9 patients across 22 imaging studies. These most commonly involved the head and face $(9 / 22,41 \%)$ followed by the neck $(3 / 22,14 \%)$. Thus, injuries of the head, face, and neck constituted $55 \%$ of concomitant injuries. 


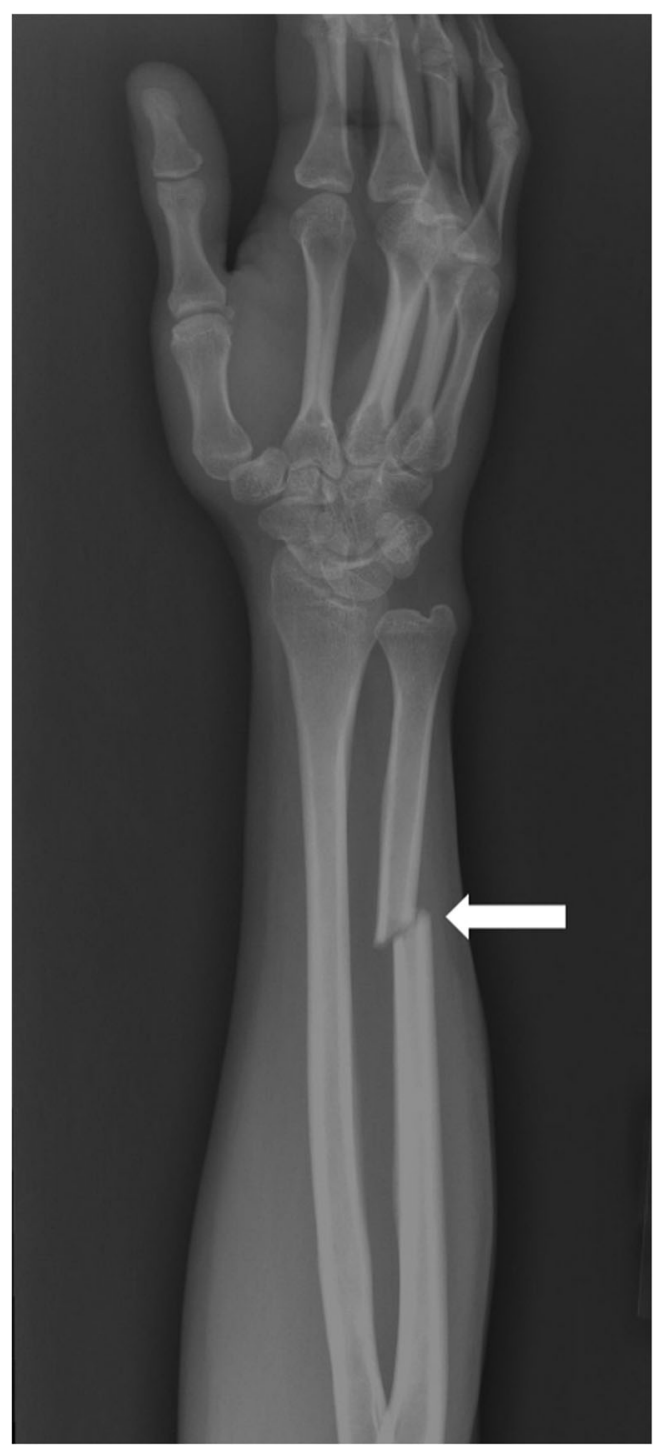

Fig. 3 Distal ulna fracture. 34-year-old Hispanic female with an unknown mechanism of injury. Oblique radiograph of the right forearm shows a mildly displaced acute fracture of the distal ulna (arrow)

\section{Non-synchronous injuries}

Twelve patients $(22 \%)$ had imaging evidence of injuries on other occasions before their first UE injury, with a total of 16 injuries. Of these, the most common was a lower extremity injury $(7 / 16,43 \%)$, followed by the head and face $(6 / 16$, $38 \%$ ). The two obstetric/gynecologic injuries noted before the first UE injury were subchorionic hematoma and intrauterine growth retardation.

The UE injury was followed by another injury at a later time, in 24 patients (44\%), with a total of 61 injuries. Of these, the most common was head and face injuries $(21 / 61,35 \%)$ followed by a recurrent UE injury $(16 / 61,26 \%)$.

Twelve patients (22\%) had recurrent UE injuries. Of these, 6 had recurrent injuries of the same hand

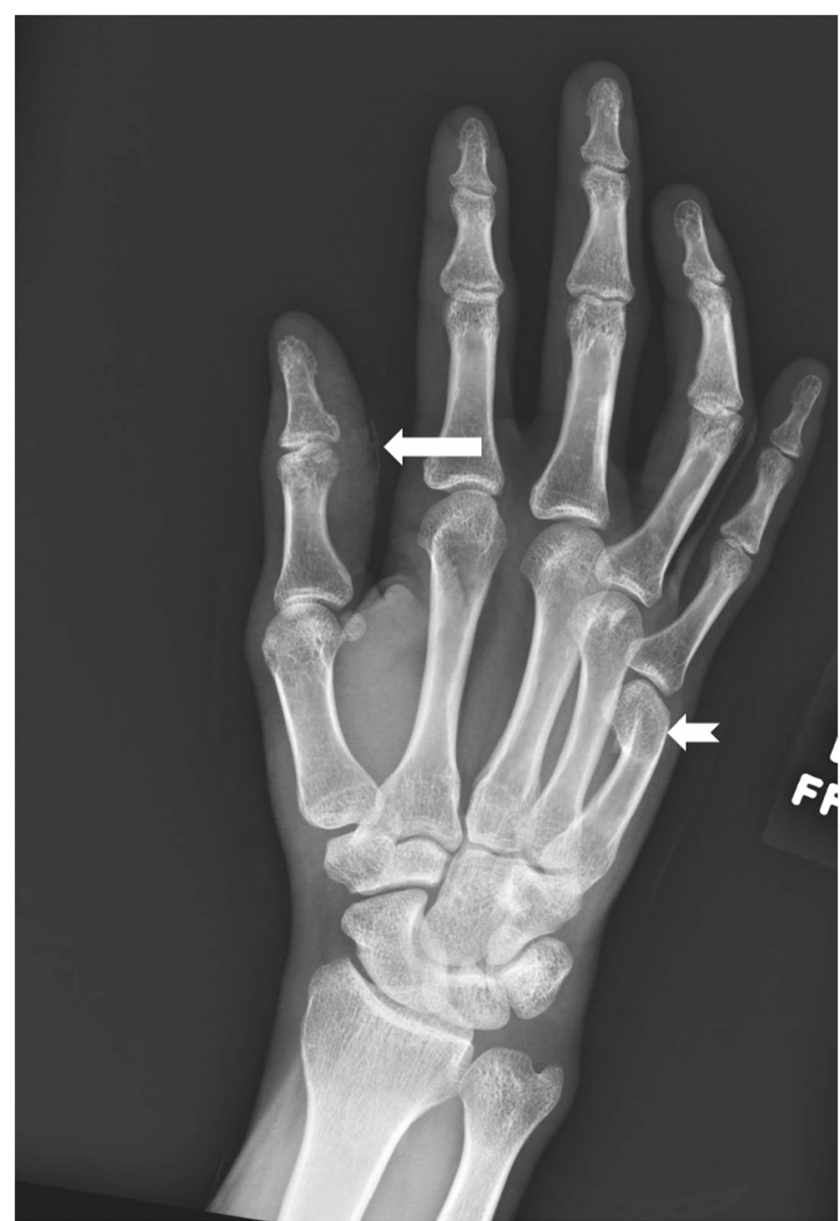

Fig. 4 Acute and chronic injuries in the same hand. 34-year-old African American male with reported history of assault by an intimate partner. Frontal radiograph of the right hand shows a right thumb laceration (straight arrow) with a chronic 5th digit metacarpal neck fracture (notched arrow)

(Fig. 5). Characteristics of the upper extremity injuries are summarized in Table 2.

\section{Discussion}

Almost half of women killed by their intimate partners present to an emergency department in the 2 years prior to their death, yet only $5-30 \%$ of IPV cases are successfully identified in the ED $[14,18]$. The results of our study support the fact that IPV continues to be profoundly underdiagnosed due to underreporting and poor documentation [4]. Only 17\% of patients in this IPV cohort disclosed IPV as the cause of their injury with IPV screening documented in only $25 \%$ of injuries. Clearly, we need to move beyond the patient's self-reporting and take an objective approach to address this highly prevalent public health issue [19]. By establishing injury patterns typical of IPV, our study seeks to help health care providers identify IPV before escalation of the violence [19]. 
Fig. 5 Recurrent injuries in the same hand. Lateral radiograph of the hand (a) in a 32-year-old African American female with an unknown mechanism of injury shows soft tissue swelling over the dorsum of the hand (arrow). The patient presented 2 years later with a history of assault by an intimate partner. Frontal radiograph of the right hand during the second presentation (b) shows an acute fracture of the middle phalanx of the fourth digit (arrow)
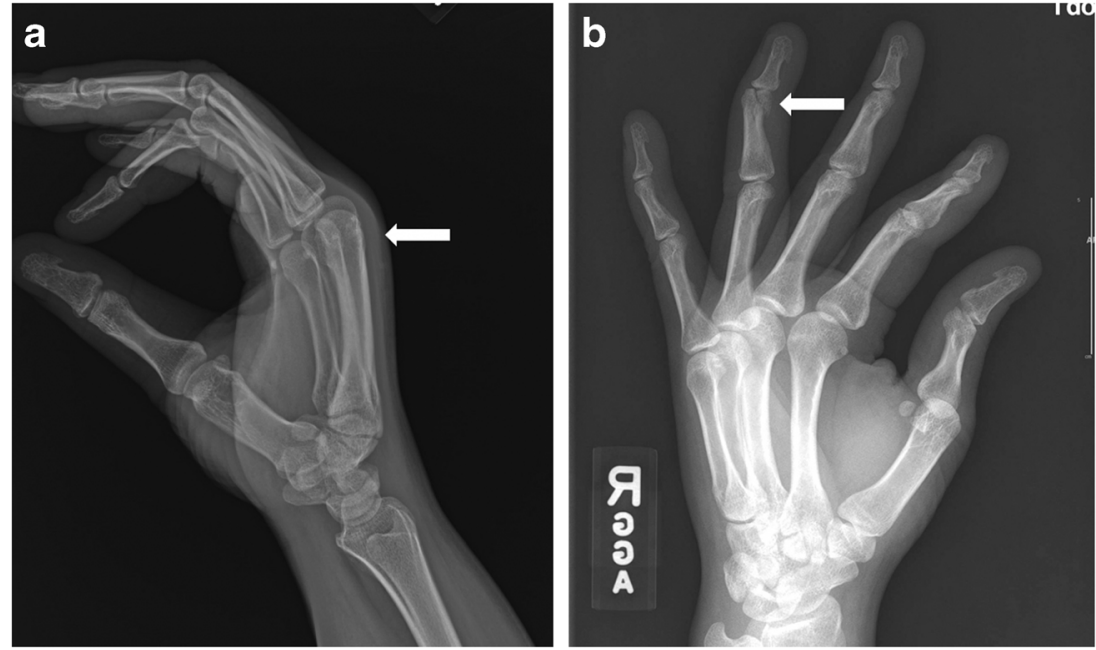

Earlier studies have identified the spectrum of all types of physical injuries in victims of IPV $[12,13,15,20]$. However, our study describes specific patterns of UE injuries in such patients and characterizes them in detail: by the site involved, by morphological features, and by the temporal associations of injuries at other sites on radiological studies to that of UE injuries. In our study, the hand and finger were the most commonly injured sites, across fractures, dislocations, and soft tissue injuries. Fractures were overall more common, and the finger was the most common site of fracture ( $46 \%$ of fractures). In contrast, in the general population, fractures of the radius and ulna are more common than fractures of the fingers [21]. Finger fractures in the general population are often related to sports injuries, getting slammed in a door or carelessness with tools, and are twice more common in men than women [22]. In our cohort of predominantly women, finger fractures are likely related to altercations.

In our study, fractures of the medial aspect of the hand and fingers constituted $26 \%$ of all UE fractures potentially as the medial aspect of the hand often receives the impact due to defensive posturing, in the setting of an assault. The distal ulna fracture has been labeled a nightstick fracture, owing to its association with blocking a strike [23]. However, only one of the medial UE injuries in our study was of the distal ulna, with the rest being of the hand. This possibly indicates that most of these episodes were altercations and did not involve the patient being struck with an object or a weapon.

Our study included 6 metacarpal fractures with all six being of the 5 th digit. These represented $21 \%$ of all hand fractures. In the general population, 5 th digit metacarpal fractures represent about $11 \%$ of all hand fractures with a lower incidence in women [24], particularly in the 20-40-year age group [25, 26]. Despite having a mostly female cohort, the high proportion of 5 th digit metacarpal fractures in our study again points to an association with assault. Of note, only $17 \%$ of upper extremity fractures were comminuted and displaced. The vast majority $(92 \%)$ of hand fractures were non-displaced and non- comminuted, potentially due to IPV altercations being associated with lower energy trauma.

A combination of acute and non-acute fractures in the same location may indicate a repetitive pattern of assault, as is often seen with IPV. In our study, non-acute fractures were seen in 4 patients. Our study also showed that one of the most common injuries to follow a UE injury was another UE injury. In more than half of these patients with repeated UE injury, the same hand was noted to be injured on successive imaging studies. We believe these findings reflect the repetitive and consistent nature of the abuse.

Multiple prior studies have noted the head, face, and neck region to be the most common site involved in IPV [13, 15, 20]. In our study, head and neck injuries constituted $55 \%$ of injuries concomitant with UE injuries and were also the most common injuries to follow a UE injury. Thus, a combination of UE (defensive) and head or face (target) injuries can indicate IPV.

Our study has some limitations. This is a retrospective, descriptive study from a single institution with a relatively small number of IPV victims with UE injuries. A causal relationship to IPV could not be assessed for each injury due to the retrospective nature of the study and the lack of reliable history documented in EHR at the time of reporting. We recognize that it is necessary to include a control population to define and validate specific imaging correlates of any pathology. However, it is virtually impossible to establish a control population for IPV, considering the significantly low proportion of patients disclosing such a history. A patient in the control group could potentially be a victim of IPV but not forthcoming. Even in our cohort of known victims, only $65 \%$ of victims had IPV documented in their charts. We believe our study provides a basis for future studies in this field to define injury patterns linked to IPV and establishing an objective basis for identifying victims, similar to pediatric non-accidental injuries. As per the recommendations of Matoori et al [27], when radiologists suspect IPV based on 
Table 2 Patterns of injuries

\begin{tabular}{|c|c|}
\hline & $N=88$ \\
\hline Side of injury & $R=50(57 \%)$ \\
\hline Hand injuries & $56(64 \%)$ \\
\hline Fracture & $N=46$ \\
\hline Arm-humerus & $3(6 \%)$ \\
\hline Forearm-radius and ulna & $15(33 \%)$ \\
\hline Wrist (carpal) & $1(2 \%)$ \\
\hline Hand (metacarpal) & $6(13 \%)$ \\
\hline Finger (phalanx) & $21(46 \%)$ \\
\hline Fracture characteristics & $N=46$ \\
\hline Comminuted or displaced & $8(17 \%)$ \\
\hline Non-acute fracture & $6(13 \%)$ \\
\hline None & $32(70 \%)$ \\
\hline Hand and finger fractures & $N=27$ \\
\hline Medial hand & $12(44 \%)$ \\
\hline Dislocation/Subluxation & $N=8$ \\
\hline IPJ & $4(50 \%)$ \\
\hline MCP & $1(12.5 \%)$ \\
\hline DRU & $1(12.5 \%)$ \\
\hline Elbow & $1(12.5 \%)$ \\
\hline $\mathrm{AC}$ & $1(12.5 \%)$ \\
\hline Soft tissue injuries & $N=34$ \\
\hline Hand and wrist & $23(68 \%)$ \\
\hline Forearm & $9(26 \%)$ \\
\hline Shoulder/arm & $2(6 \%)$ \\
\hline Concomitant injury & $N=22$ \\
\hline Pelvis & $2(9 \%)$ \\
\hline Head and face & $9(41 \%)$ \\
\hline Neck & $3(14 \%)$ \\
\hline Chest & $3(14 \%)$ \\
\hline Abdomen & $1(4 \%)$ \\
\hline Lower extremity & $3(14 \%)$ \\
\hline Spine & $1(4 \%)$ \\
\hline Injuries following UE injury & $N=61$ \\
\hline Upper extremity & $16(26 \%)$ \\
\hline Lower extremity & $7(11 \%)$ \\
\hline Pelvis/hip & $2(3 \%)$ \\
\hline Thorax & $8(13 \%)$ \\
\hline Head and face & $21(35 \%)$ \\
\hline Abdomen & $2(3 \%)$ \\
\hline Spine & $3(5 \%)$ \\
\hline Neck & $2(3 \%)$ \\
\hline Injuries preceding UE injury & $N=16$ \\
\hline Head and face & $6(38 \%)$ \\
\hline Thorax & $1(6 \%)$ \\
\hline Lower extremity & $7(43 \%)$ \\
\hline Obstetric & $2(13 \%)$ \\
\hline
\end{tabular}

$I P J$ interphalangeal joint, $M C P$ metacarpophalangeal joint, $D R U$ distal radioulnar joint, $A C$ acromioclavicular joint, $U E$ upper extremity imaging findings, they must alert the treating physician which should lead to a confidential conversation with the patient. If the patient confirms IPV, standard institutional practice must be followed, and if the patient denies IPV, he/she can be offered actionable resources in the event of a future need [27].

\section{Conclusion}

IPV is a significant public health issue worldwide. The radiologist can play a crucial role in identification and documentation similar to non-accidental trauma in children; however, the radiological patterns need to be studied and established first. By reviewing upper extremity injuries in a known cohort of IPV, we recognized that hand injuries are the most common type of UE injuries in patients with IPV. Notably, our study found that the finger is the most common site of the fracture, and the 5th digit is the most common region of the hand fracture. Repeated injuries involving the same location, especially in the hand, and a combination of medial hand and head or face injuries, could indicate IPV. Early detection can facilitate early diagnosis, thereby preventing life-threatening injuries.

\section{Funding}

The study was funded in part by:

1. Gillian Reny Stepping Strong for Trauma Innovation

2. Partners Innovation Discovery Grant Award

3. Brigham Care Redesign Incubator and Startup Program

\section{Compliance with ethical standards}

Guarantor The scientific guarantor of this publication is Bharti Khurana MD.

Conflict of interest The authors of this manuscript declare no relationships with any companies whose products or services may be related to the subject matter of the article.

Statistics and biometry No complex statistical methods were necessary for this paper.

Informed consent Written informed consent was waived by the Institutional Review Board.

Ethical approval Institutional Review Board approval was obtained.

Methodology

- Retrospective

- Cross sectional study

- Performed at one institution

\section{References}

1. Violence against women. https://www.who.int/news-room/factsheets/detail/violence-against-women. Accessed 10 May 2020

2. Sharon G Smith, Xinjian Zhang, Kathleen C Basile, et al (2019) 2015 NISVS Data Brief|Violence Prevention|Injury Center|CDC. 
https://www.cdc.gov/violenceprevention/datasources/nisvs/ 2015NISVSdatabrief.html. Accessed 13 May 2020

3. Dicola D, Spaar E (2016) Intimate partner violence. Am Fam Physician 94:646-651

4. Gracia E (2004) Unreported cases of domestic violence against women: towards an epidemiology of social silence, tolerance, and inhibition. J Epidemiol Community Health 58:536-537. https://doi. org/10.1136/jech.2003.019604

5. Elliott L, Nerney M, Jones T, Friedmann PD (2002) Barriers to screening for domestic violence. J Gen Intern Med 17:112-116. https://doi.org/10.1046/j.1525-1497.2002.10233.x

6. Rose D, Trevillion K, Woodall A, Morgan C, Feder G, Howard L (2011) Barriers and facilitators of disclosures of domestic violence by mental health service users: qualitative study. Br J Psychiatry J Ment Sci 198:189-194. https://doi.org/10.1192/bjp.bp.109.072389

7. China's Hidden Epidemic: Domestic Violence. https://thediplomat. com/2020/04/chinas-hidden-epidemic-domestic-violence/. Accessed 23 May 2020

8. Domestic violence calls increase amid coronavirus lockdown, police say. In: NBC News. https://www.nbcnews.com/news/us-news/ police-see-rise-domestic-violence-calls-amid-coronaviruslockdown-n1176151. Accessed 23 May 2020

9. Chandan JS, Taylor J, Bradbury-Jones C, Nirantharakumar K, Kane E, Bandyopadhyay S (2020) COVID-19: a public health approach to manage domestic violence is needed. Lancet Public Health. https://doi.org/10.1016/S2468-2667(20)30112-2

10. Gosangi B, Park H, Thomas R, et al (2020) Exacerbation of physical intimate partner violence during COVID-19 lockdown. Radiology 202866. https://doi.org/10.1148/radiol.2020202866

11. Matoori S, Khurana B, Balcom MC et al (2020) Intimate partner violence crisis in the COVID-19 pandemic: how can radiologists make a difference? Eur Radiol. https://doi.org/10.1007/s00330020-07043-w

12. George E, Phillips $\mathrm{CH}$, Shah N, et al (2019) Radiologic findings in intimate partner violence. Radiology 291:62-69. https://doi.org/10. 1148/radiol.2019180801

13. Loder RT, Momper L (2020) Demographics and fracture patterns of patients presenting to US emergency departments for intimate partner violence. J Am Acad Orthop Surg Glob Res Rev 4:e20.00009. https://doi.org/10.5435/JAAOSGlobal-D-20-00009

14. Wadman MC, Muelleman RL (1999) Domestic violence homicides: ED use before victimization. Am J Emerg Med 17:689691. https://doi.org/10.1016/S0735-6757(99)90161-4

15. Bhandari M, Dosanjh S, Tornetta P, Matthews D (2006) Musculoskeletal manifestations of physical abuse after intimate partner violence. J Trauma 61:1473-1479. https://doi.org/10. 1097/01.ta.0000196419.36019.5a

16. Le BT, Dierks EJ, Ueeck BA, Homer LD, Potter BF (2001) Maxillofacial injuries associated with domestic violence. J Oral
Maxillofac Surg 59:1277-1283; discussion 1283-1284. https://oi. org/10.1053/joms.2001.27490

17. Wong JY, Choi AW, Fong DY, Wong JK, Lau CL, Kam CW (2014) Patterns, aetiology and risk factors of intimate partner violence-related injuries to head, neck and face in Chinese women. BMC Womens Health 14:6. https://doi.org/10.1186/1472-687414-6

18. Reis BY, Kohane IS, Mandl KD (2009) Longitudinal histories as predictors of future diagnoses of domestic abuse: modelling study. BMJ 339. https://doi.org/10.1136/bmj.b3677

19. Khurana B, Seltzer SE, Kohane IS, Boland GW (2020) Making the thevisibleiblefuture diagnoses of domestic abuse: modelling study. BMJ Qual Saf 29:241-244. https://doi.org/10.1136/bmjqs-2019009905

20. Wu V, Huff H, Bhandari M (2010) Pattern of physical injury associated with intimate partner violence in women presenting to the emergency department: a systematic review and meta-analysis. Trauma Violence Abuse 11:71-82. https://doi.org/10.1177/ 1524838010367503

21. Chung KC, Spilson SV (2001) The frequency and epidemiology of hand and forearm fractures in the United States. J Hand Surg 26: 908-915. https://doi.org/10.1053/jhsu.2001.26322

22. Anakwe RE, Aitken SA, Cowie JG, Middleton SD, Court-Brown CM (2011) The epidemiology of fractures of the hand and the influence of social deprivation. J Hand Surg Eur Vol 36:62-65. https://doi.org/10.1177/1753193410381823

23. Lee P, Hunter TB, Taljanovic M (2004) Musculoskeletal colloquialisms: how did we come up with these names? Radiographics 24: 1009-1027. https://doi.org/10.1148/rg.244045015

24. Van Onselen EBH, Karim RB, Hage JJ, Ritt MJPF (2003) Prevalence and distribution of hand fractures. J Hand Surg 28: 491-495. https://doi.org/10.1016/S0266-7681(03)00103-7

25. de Jonge JJ, Kingma J, van der Lei B, Klasen HJ (1994) Fractures of the metacarpals. A retrospective analysis of incidence and aetiology and a review of the English-language literature. Injury 25:365-369. https://doi.org/10.1016/0020-1383(94)90127-9

26. Nakashian MN, Pointer L, Owens BD, Wolf JM (2012) Incidence of metacarpal fractures in the US population. Hand (N Y) 7:426430. https://doi.org/10.1007/s11552-012-9442-0

27. Matoori S, Khurana B, Balcom MC et al (2020) Addressing intimate partner violence during the COVID-19 pandemic and beyond: how radiologists can make a difference. Eur Radiol:1-6. https://doi. org/10.1007/s00330-020-07332-4

Publisher's note Springer Nature remains neutral with regard to jurisdictional claims in published maps and institutional affiliations. 